\title{
Correlation of active contact location with weight gain after subthalamic nucleus deep brain stimulation: a case series
}

\author{
Katsuki Eguchi ${ }^{*}$, Shinichi Shirai ${ }^{1}$, Masaaki Matsushima', Takahiro Kano ${ }^{1}$, Kazuyoshi Yamazaki $^{2}$, Shuji Hamauchi $^{2}$, \\ Toru Sasamori ${ }^{3}$, Toshitaka Seki ${ }^{2}$, Kenji Hirata ${ }^{4}$, Mayumi Kitagawa ${ }^{5}$, Mika Otsuki ${ }^{6}$, Tohru Shiga ${ }^{7}$, Kiyohiro Houkin², \\ Hidenao Sasaki ${ }^{1}$ and Ichiro Yabe ${ }^{1}$
}

\begin{abstract}
Background: Weight gain (WG) is a frequently reported side effect of subthalamic deep brain stimulation; however, the underlying mechanisms remain unclear. The active contact locations influence the clinical outcomes of subthalamic deep brain stimulation, but it is unclear whether WG is directly associated with the active contact locations. We aimed to determine whether WG is associated with the subthalamic deep brain stimulation active contact locations.

Methods: We enrolled 14 patients with Parkinson's disease who underwent bilateral subthalamic deep brain stimulation between 2013 and 2019. Bodyweight and body mass index were measured before and one year following the surgery. The Lead-DBS Matlab toolbox was used to determine the active contact locations based on magnetic resonance imaging and computed tomography. We also created sweet spot maps for WG using voxelwise statistics, based on volume of tissue activation and the WG of each patient. Fluorodeoxyglucose-positron emission tomography data were also acquired before and one year following surgery, and statistical parametric mapping was used to evaluate changes in brain metabolism. We examined which brain regions' metabolism fluctuation significantly correlated with increased body mass index scores and positron emission tomography data.

Results: One year after surgery, the body mass index increase was $2.03 \mathrm{~kg} / \mathrm{m}^{2}$. The sweet spots for WG were bilateral, mainly located dorsally outside of the subthalamic nucleus (STN). Furthermore, WG was correlated with increased metabolism in the left limbic and associative regions, including the middle temporal gyrus, inferior frontal gyrus, and orbital gyrus.

Conclusions: Although the mechanisms underlying WG following subthalamic deep brain stimulation are possibly multifactorial, our findings suggest that dorsal stimulation outside of STN may lead to WG. The metabolic changes in limbic and associative cortical regions after STN-DBS may also be one of the mechanisms underlying WG. Further studies are warranted to confirm whether dorsal stimulation outside of STN changes the activities of these cortical regions.
\end{abstract}

Keywords: Deep brain stimulation, Parkinson's disease, Subthalamic nucleus, Weight gain

\footnotetext{
*Correspondence: k198762@gmail.com

'Department of Neurology, Faculty of Medicine, Graduate School of

Medicine, Hokkaido University, Kita 15, Nishi 7, Kita-ku, 060-8638 Sapporo,

Japan

Full list of author information is available at the end of the article
}

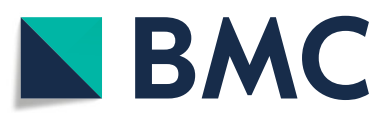

(- The Author(s). 2021 Open Access This article is licensed under a Creative Commons Attribution 4.0 International License, which permits use, sharing, adaptation, distribution and reproduction in any medium or format, as long as you give appropriate credit to the original author(s) and the source, provide a link to the Creative Commons licence, and indicate if changes were made. The images or other third party material in this article are included in the article's Creative Commons licence, unless indicated otherwise in a credit line to the material. If material is not included in the article's Creative Commons licence and your intended use is not permitted by statutory regulation or exceeds the permitted use, you will need to obtain permission directly from the copyright holder. To view a copy of this licence, visit http://creativecommons.org/licenses/by/4.0/. The Creative Commons Public Domain Dedication waiver (http://creativecommons.org/publicdomain/zero/1.0/) applies to the data made available in this article, unless otherwise stated in a credit line to the data. 


\section{Background}

Subthalamic nucleus deep brain stimulation (STN-DBS) is an established and effective treatment strategy for advanced Parkinson's disease (PD) [1]. However, STN-DBS is associated with several adverse effects, of which, weight gain (WG) is one of the most common, although the underlying mechanisms have not been fully elucidated. Previous reports have suggested that WG is associated with lowered resting energy needs [2, 3], fewer motor complications (especially dyskinesia) [4-6], changes in eating behaviors [7, 8], and hormonal factors $[9,10]$. However, these factors do not completely explain WG, which suggests that WG following STN-DBS is a multifactorial process [11].

The locations of electrodes affect the clinical outcomes and adverse effects of STN-DBS [12, 13]. Based on animal studies $[14,15]$ and studies examining the human brain using diffusion tensor imaging [16-18], the STN is divided into three functional subregions: the sensorimotor, associative, and limbic regions. Previous studies have suggested that superior motor improvement is achieved by stimulating the sensorimotor area, which is located in the dorsolateral part of the STN and is linked to the primary motor and supplementary motor cortices [17, 19-21]. Nevertheless, an increased risk of neuropsychiatric side effects may be associated with stimulation of the limbic area, which is present in the anteromedial part of the STN $[17,22]$. However, few studies have evaluated whether WG is directly correlated with the stimulation location during STN-DBS. Therefore, this study prospectively evaluated patients who underwent STN-DBS for PD and aimed to determine whether WG was correlated with the positions of the active contacts and distribution of volume of tissue activation (VTA).

In addition, several previous studies using fluorodeoxyglucose-positron emission tomography (FDG-PET) have shown a correlation between behavioral or cognitive changes and metabolic changes in specific brain regions following STN-DBS [23, 24]. We hypothesized that regions in which metabolic changes are seen, could help identify the regions in the STN correlated with WG after STN-DBS. Therefore, we also evaluated whether WG was associated with altered glucose metabolism in specific brain regions.

\section{Methods}

\section{Patients}

This prospective study recruited patients with PD, who underwent bilateral STN-DBS surgery, at Hokkaido University Hospital between 2013 and 2019. PD diagnoses were based on the UK Parkinson's Disease Society Brain Bank criteria [25]. We recruited 16 patients with PD who underwent bilateral STN-DBS surgery; we then excluded one patient who withdrew from participating and another who required electrode removal due to an infection. Thus, this study analyzed data from 14 patients, including 12 women. The median age at DBS surgery was 62.5 (55.5-68) years, and the median disease duration at DBS surgery was $14.3(12-20.5)$ years. The eligibility for DBS surgery was determined based on the guidelines of the International Parkinson and Movement Disorders Society [26]. All patients received bilaterally implanted quadripolar (from contact number '0' for the most ventral contact to ' 3 ' for the most dorsal one) DBS electrodes (3387; Medtronic, Minneapolis, MN, USA).

This study was conducted following the 1964 Declaration of Helsinki and its later amendments and was approved by the ethics panel of the institutional review board of Hokkaido University Hospital. All patients provided written informed consent before their inclusion in the study.

\section{Clinical assessment}

Clinical assessments were conducted at the preoperative baseline and one year following surgery. Bodyweight and height were measured and used to calculate the body mass index (BMI, $\left.\mathrm{kg} / \mathrm{m}^{2}\right)$. Motor symptoms were assessed using the Unified Parkinson's Disease Rating Scale (UPDRS) part III in the MedOff state at baseline and in the MedOff and DBS-on state at one year following surgery. Dyskinesia was also evaluated using items 32 and 33 of the UPDRS part IV. Neuropsychological evaluations were based on the Mini Mental State Examination (MMSE), Frontal Assessment Battery (FAB), Apathy Scale [27], and Patient Health Questionnaire-9 [28]. The levodopa-equivalent daily dose (LEDD) [29] was calculated at both baseline and one year following DBS surgery. Brain metabolism was evaluated using FDG-PET at baseline and one year following surgery. The DBS stimulation parameters were recorded at one year following surgery and included the stimulation voltage, pulse width, frequency, and active contact location.

\section{Assessing the active contact positions}

The active contact positions were evaluated using LeadDBS, which is a validated Matlab toolbox [30, 31] that was implemented using Matlab 2019b (MathWorks, Natick, MA, USA). Preoperative results were obtained via T2-weighted magnetic resonance imaging (MRI, slice thickness: $1 \mathrm{~mm}$; echo time: $222 \mathrm{~ms}$; repetition time: $2,000 \mathrm{~ms}$ ), and postoperative results were obtained via computed tomography (CT). The preoperative MRI and postoperative CT images were co-registered using advanced normalization tools [32] and then nonlinearly normalized into Montreal Neurological Institute (MNI) standard space (MNI_ICBM_2009b_NLIN_Asym). The DBS electrodes were automatically reconstructed using 
the TRAC/CORE algorithm [31] and manually refined to evaluate their coordinates in MNI space. If more than one contact was used for the stimulation, the mean coordinates of all active contacts were recorded. The positional relationships between the active contacts and the STN were assessed using the DISTAL atlas [33], which is a composite atlas based on histology, structural connectivity, and manual segmentations of a multimodal brain template normalized in MNI space.

\section{Calculation of volume of tissue activation}

VTA calculations were performed using a finite element method approach [34], implemented in Lead-DBS based on the stimulation settings of each patient. The spread of the electric field was estimated for homogenous tissue with a conductivity of $0.14 \mathrm{~S} / \mathrm{m}$. The threshold of VTA was at the electric field isolevel of $0.2 \mathrm{~V} / \mathrm{mm}$.

\section{Creation of sweet spot map for WG}

In this study, we assessed the sweet spot for WG after DBS using Lead-Group [35]. Each voxel of each patient's VTA was assigned an increase in BMI. Subsequently, all VTAs were pooled across patients and the mean increase in BMI was obtained for each voxel. To identify voxels with a significantly higher increase in BMI than the average increase of BMI of all VTAs that did not stimulate that particular voxel, a two-sample t-test was performed. This test yielded a t-statistic for every voxel that was then displayed as 3D statistical maps, which we referred to as sweet spot maps. Significant voxels (uncorrected, $\mathrm{p}<0.05$ ) were visualized on sweet spot maps for WG. Only those voxels that were at least covered by $\mathrm{n}=3$ VTAs were considered in this analysis.

\section{FDG-PET acquisition and preprocessing}

PET data were obtained using either a GEMINI TF64 (Philips, Amsterdam, Netherlands) PET-CT scanner or a Biograph 64 (Siemens, Munich, Germany) TruePoint PET-CT scanner for different patients. Time-of-flight technology was used with GEMINI TF64 but not with Biograph 64. The manufacturer of the DBS system warns that stimulation intensity may increase during $\mathrm{CT}$ and recommends turning off stimulation during the scan. In our institution, turning off DBS stimulation is required during scanning with a PET-CT scanner to avoid an increase in stimulation intensity and incurring any side effects. Therefore, in this study, a PET was performed in the MedOn at baseline and in the MedOn and DBS-off state post-operation. DBS stimulation was turned off 30-60 min before PET. Patients were instructed to fast overnight before PET [18]. F-FDG (4.5 MBq/kg) was administered intravenously, and serum glucose was measured to exclude the patients showing fasting hyperglycemia (>150 mg/dL). The images were acquired
60 min following FDG administration for 10-min emission scanning. Images were reconstructed using ordered subset expectation-maximization. The PET images were preprocessed using statistical parametric mapping (SPM12; Wellcome Department of Cognitive Neurology, London, UK) on Matlab 2019b. The PET images were initially subjected to affine and nonlinear spatial normalization into the MNI brain space, although we used an FDG-PET-specific template that was described by Della Rosa et al. [36] instead of the default $\mathrm{H}_{2} \mathrm{O}-\mathrm{SPM}$ template [15]. The normalized images were then smoothed using an 8-mm isotropic Gaussian filter to compensate for individual anatomical variability. Finally, we created a percent signal change map (PSC map) using the following formula:

$$
\mathrm{PSC}=\left(\mathrm{V}_{l \mathrm{y}}-\mathrm{V}_{\mathrm{b}}\right) \times 100 / \mathrm{V}_{\mathrm{b}}
$$

where, $V_{1 y}$ and $V_{b}$ represent voxel values at one year following surgery and baseline, respectively.

\section{Statistical analysis}

Scores are reported as the median \pm interquartile range (IQR), and nonparametric analyses were used based on the small sample size. Preoperative and postoperative values for BMI, UPDRS part III and IV scores, neuropsychological data, and LEDD values were compared using the Wilcoxon signed-rank test, and $\mathrm{p}<0.001$ was considered as statistically significant using Bonferroni correction. Stimulation voltage and active contact coordinates for the left and right sides were compared using Wilcoxon's rank-sum test. A multivariate regression analysis was performed with WG as the dependent variable and contacts' coordinates that showed a correlation with WG, age, body weight before surgery, sex, stimulation voltage, frequency, pulse width, dyskinesia reduction (scores of UPDRS part IV items $32+33$ ), and LEDD reduction as independent variables to adjust for any possible confounders $(\mathrm{p}<0.05$ was considered as statistically significant). Statistical analyses were carried out using JMP Pro software (version 14; SAS Inc., Cary, North Carolina, USA).

The PET data were analyzed using SPM12 (Wellcome Department of Cognitive Neurology, London, UK). To identify which brain regions' metabolism change correlated significantly with increased BMI scores, a general linear model was tested at each voxel with the BMI score as a covariate using a PSC map. LEDD was included in the SPM analysis as a covariate. A voxel-level threshold of $P<0.05$ (family-wise error corrected for multiple comparisons) was used to assess the SPM $t$ values. If statistical significance was not reached, we performed the same analysis with a voxel-level threshold of 
$P<0.005$ (uncorrected for multiple comparisons), considering the small sample size and the study's exploratory nature. Only clusters containing $>100$ voxels were reported.

\section{Results}

\section{Clinical outcomes}

Table 1 shows the clinical values at preoperative baseline and one year following DBS surgery. A significant motor improvement was observed one year post-surgery, based on the decreased UPDRS part III scores in the MedOff state. A non-significant trend toward decreased values for items 32 and 33 of the UPDRS part IV was also observed. Bodyweight and BMI values increased significantly following STN-DBS. The neuropsychological assessments revealed a small but statistically significant increase in the FAB score but no significant changes in the other scales. There was a non-significant trend of decrease in LEDD after DBS surgery.

\section{Stimulation parameters and active contact positions} Each patient's stimulation parameters at one year following DBS surgery are shown in Table 2. The stimulation intensities were similar between the right and left sides. Figure 1 shows the electrode locations for the STN of all patients, as defined using the DISTAL atlas and MNI space. The median coordinates of the right-side active contacts were $12.5(11.7-13.5) \mathrm{mm}$ on the $\mathrm{X}$-axis, -14.5 $(-13.5$ to -14.9$) \mathrm{mm}$ on the $\mathrm{Y}$-axis, and $-6.4(-5.6$ to $-7.6) \mathrm{mm}$ on the $\mathrm{Z}$-axis. The mean left-side coordinates were $-13.6(-11.5$ to -14.2$) \mathrm{mm}$ on the $\mathrm{X}$-axis, -13.6 $(-11.8$ to -15.6$) \mathrm{mm}$ on the $\mathrm{Y}$-axis, and $-6.0(-5.1$ to -6.8) $\mathrm{mm}$ on the Z-axis. The $\mathrm{X}-, \mathrm{Y}-$, and $\mathrm{Z}$-axis coordinates were not significantly different between the right and left sides, although the left-side $\mathrm{Y}$-axis coordinates had a larger IQR than the right-side Y-axis coordinates.

\section{Sweet spot map for WG}

Figure 2 shows the sweet spot map for the WG results of voxel-wise statistical analysis using VTA. The sweet spots for WG were mainly located dorsally and outside of the STN, bilaterally.

\section{Correlations with increased BMI}

We could not obtain a statistically significant model on multivariate regression analysis $(\mathrm{P}=0.11)$.

\section{PET image analysis}

We analyzed PET data from 13 patients since one patient's PET data were missing. A GEMINI TF64 PETCT scanner was used for 12 patients, while a Biograph 64 TruePoint PET-CT scanner was used for the remaining patient. None of the voxels were significant at a voxel-level threshold of $P<0.05$ (family-wise error corrected for multiple comparisons). However, at a threshold of $P<0.005$ (uncorrected for multiple comparisons), we identified several clusters with positive correlations between WG and increased metabolism in the left hemisphere (Table 3; Figs. 3 and 4). The correlations were observed in the left middle temporal gyrus, inferior frontal gyrus, lateral orbital gyrus, anterior orbital gyrus, and planum polare. We did not observe any negative correlations between WG and brain metabolism in these various areas.

\section{Discussion}

This study showed a mean BMI increase of $2 \mathrm{~kg} / \mathrm{m}^{2}$ at 1 year following DBS surgery; this concurs with previous findings [37]. Furthermore, the mean active contact coordinates in our study were similar to the preferred coordinates for motor improvement in previous studies [38]. The sweet spot for WG was located in the dorsal part of the STN and dorsally outside the STN on both sides. We could not detect a significant

Table 1 Clinical values at baseline and 1-year follow-up

\begin{tabular}{lllc}
\hline & Baseline & 1-year Follow-up & $p$-value \\
\hline Bodyweight & $55.8(49.7-63.6)$ & $60.7(53.1-69.6)$ & $<0.001$ \\
BMI & $23.1(20.1-25.7)$ & $24.5(22.0-29.1)$ & $<0.001$ \\
UPDRS part III (MedOn) & $14(10-18)$ & $8(4.5-19.3)$ & 0.13 \\
UPDRS part III (MedOff) & $40(33.8-44.3)$ & $16(11.8-23)$ & $<0.001$ \\
UPDRS part IV (items 32+33) & $1.5(0-4)$ & $1(0-2.25)$ & 0.12 \\
MMSE & $29(27.8-30)$ & $29(28-30)$ & $15.5(13.8-17.3)$ \\
FAB & $14.5(14.5-17.5)$ & $14(4.8-16.3)$ & 0.3 \\
Apathy scale & $13.5(7.5-16)$ & $4(0-7.8)$ & 0.023 \\
PHQ-9 & $5(4-8)$ & $418.5(297.3-656.3)$ & 0.47 \\
LEDD & $689(363-748.5)$ & & 0.43 \\
\hline
\end{tabular}

Scores are reported as the median (interquartile range)

$B M I$ body mass index; FAB Frontal Assessment Battery; LEDD levodopa-equivalent daily dose; MedOff a condition without intaking medication; MedOn a condition requiring intaking medication. MMSE Mini Mental State Examination; PHQ-9 Patient Health Questionnaire-9; UPDRS Unified Parkinson's Disease Rating Scale 
Table 2 BMI change from baseline and stimulation parameters at one year following surgery

\begin{tabular}{|c|c|c|c|c|c|c|c|c|c|c|}
\hline \multirow[b]{2}{*}{ Subject No. } & \multirow[t]{2}{*}{ Disease duration at DBS surgery (year) } & \multirow[t]{2}{*}{$\begin{array}{l}\text { Change of BMI } \\
\text { from baseline }\end{array}$} & \multicolumn{2}{|c|}{ Active contacts } & \multicolumn{2}{|c|}{$\begin{array}{l}\text { Frequency } \\
(\mathrm{Hz})\end{array}$} & \multicolumn{2}{|c|}{$\begin{array}{l}\text { Stimulation } \\
\text { Intensity (V) }\end{array}$} & \multicolumn{2}{|c|}{$\begin{array}{l}\text { Pulse width } \\
(\mu \mathrm{s})\end{array}$} \\
\hline & & & Right & Left & Right & Left & Right & Left & Right & Left \\
\hline 1 & 8 & 2.3 & $C(+) 1(-) 2(-)$ & $C(+) 2(-)$ & 130 & 130 & 1.5 & 2.2 & 60 & 60 \\
\hline 2 & 18 & 3.8 & $C(+) 1(-)$ & $C(+) 2(-)$ & 130 & 130 & 1.2 & 1.2 & 60 & 60 \\
\hline 3 & 23 & 3.2 & $C(+) 1(-) 2(-)$ & $C(+) 2(-)$ & 130 & 130 & 2.6 & 1.8 & 60 & 60 \\
\hline 4 & 8 & 1.0 & $C(+) 3(-)$ & $C(+) 3(-)$ & 130 & 130 & 1 & 1 & 60 & 60 \\
\hline 5 & 9 & -0.2 & $C(+) 1(-)$ & $C(+) 1(-)$ & 130 & 130 & 1 & 2 & 60 & 60 \\
\hline 6 & 13 & -0.2 & $C(+) 2(-)$ & $0(+) 1(-)$ & 130 & 130 & 2.8 & 3.2 & 60 & 150 \\
\hline 7 & 13 & 4.6 & $C(+) 2(-)$ & $C(+) 2(-)$ & 60 & 60 & 2.2 & 3.7 & 60 & 90 \\
\hline 8 & 14 & 1.3 & $1(+) 2(-)$ & $C(+) 2(-)$ & 130 & 130 & 3.4 & 3.2 & 150 & 90 \\
\hline 9 & 13 & 2.8 & $C(+) 2(-)$ & $C(+) 3(-)$ & 130 & 130 & 3 & 3 & 60 & 60 \\
\hline 10 & 15 & 3.7 & $C(+) 2(-)$ & $C(+) 2(-)$ & 130 & 130 & 2.8 & 2.8 & 90 & 90 \\
\hline 11 & 6 & 0.7 & $C(+) 2(-)$ & $C(+) 2(-)$ & 130 & 130 & 1.4 & 1.4 & 90 & 90 \\
\hline 12 & 11 & 2.4 & $C(+) 2(-)$ & $C(+) 2(-)$ & 130 & 130 & 2.1 & 1.7 & 60 & 60 \\
\hline 13 & 8 & 2.3 & $C(+) 1(-)$ & $C(+) 2(-)$ & 130 & 130 & 1.8 & 3 & 60 & 60 \\
\hline 14 & 8 & 0.7 & $C(+) 2(-)$ & $C(+) 1(-)$ & 130 & 130 & 1.9 & 2 & 60 & 60 \\
\hline
\end{tabular}

correlation between WG and dyskinesia reduction; previous findings using multivariate regression analysis remain conflicting $[4-6,38]$.

In this study, sweet spots for WG were located in both, the dorsal part of the STN and dorsal to the STN on both sides. These findings agree with those of a

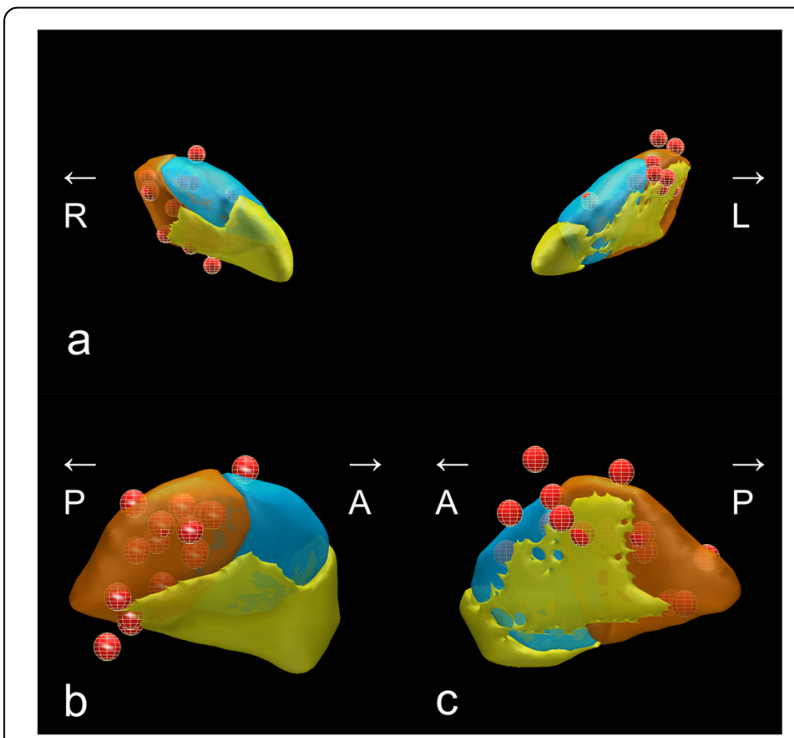

Fig. 1 Meshed red points represent positions of active contacts. (a) Anterior view of the bilateral lead locations for all 14 patients as well as the subthalamic nucleus (STN), as defined using the DISTAL atlas in Montreal Neurological Institute space [33]. The functional

subregions of the STN are highlighted (sensorimotor STN in copper, associative STN in blue, and limbic STN in yellow). The lateral views are shown for the right STN $(\mathbf{b})$ and the left STN $(\mathbf{c})$. This figure was created using Lead Group [35]. R, right; L, left; A, anterior; P, posterior

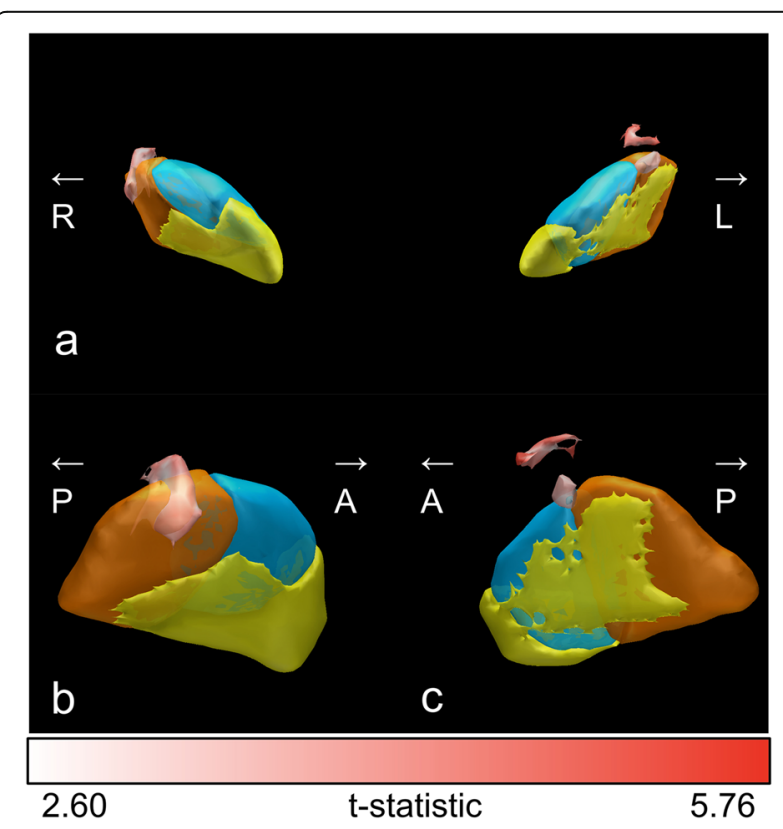

Fig. 2 The sweet spot map for weight gain based on the results of the voxel-wise statistical analysis. (a), (b), and (c) show the anterior view of the bilateral section of the STN, lateral view of right STN, and lateral view of left STN, respectively. The functional subregions of the STN are highlighted (sensorimotor STN in copper, associative STN in blue, and limbic STN in yellow). The color bar shows tstatistic of the sweet spot map. This figure was created using Lead Group [35]. R, right; L, left; A, anterior; $P$, posterior 
Table 3 Correlations between weight gain and increased brain metabolism at various locations

\begin{tabular}{llllll}
\hline & Coordinates & & & \\
\hline Region & $X$ & $Y$ & Peak T value & No. of Voxels \\
Left middle temporal gyrus & -68 & -16 & -20 & 6.8 & 575 \\
Left triangular part of the inferior frontal gyrus & -34 & 32 & 2 & 6.26 & 401 \\
Left lateral orbital gyrus & -44 & 32 & -16 & 4.83 & 4.11 \\
Left anterior orbital gyrus & -32 & 62 & -10 & -8 & 103 \\
Left planum polare & -42 & -12 & -8 & 107 \\
\hline
\end{tabular}

The uncorrected thresholds were significant $(P<0.005)$ at the voxel level. Only clusters with $>100$ voxels are reported

previous report, which indicated that active contacts located in the zona incerta (dorsal to the STN) were correlated with increased appetite after STN-DBS [39]. The zona incerta contains neurons expressing melaninconcentrating hormone, which is involved in the regulation of feeding [40]. Thus, our finding of the location of the sweet spot for WG may be explained by the stimulation of the zona incerta and neurons that express melanin-concentrating hormone.

We observed correlations between WG and increased metabolism in several brain regions that belonged to the limbic and associative areas. This finding may explain another mechanism underlying WG following STNDBS. The STN plays an important role in reward processing [41], and several studies have indicated that the STN is involved in controlling appetite and eating behaviors. For instance, a study on non-human primates illustrated that STN activity increased during food reward anticipation and delivery [42]. In humans, stroke or tumors affecting the STN causes hyperphagia and increases appetite [43, 44], and abnormal eating behaviors have been reported following STN-DBS [7, 45-49]. These findings from previous studies suggest that behavioral changes after STN-DBS may cause WG. A previous FDG-PET study showed that WG after STN-DBS was correlated with increased metabolism in the limbic and associative regions, including the orbitofrontal cortex, lateral and medial parts of the temporal lobe, anterior cingulate cortex, and retrosplenial cortex [38]. Other PET and functional MRI studies have also suggested that a broad network of limbic and paralimbic structures mediate the desire for food [50-57]. This network is thought to integrate sensory information with the cognitive desire for food, and induces behaviors that aim to obtain food $[58,59]$. Regions with increased brain metabolism in our study were also associated with the processing of desire for food; changes of the activities in the limbic and associative areas may modify food-related behavior, ultimately causing WG. However, our sweet spot map for WG in this study did not contain the limbic STN, which has strong structural connectivity with the limbic brain region. The hyperdirect pathways, connecting the cortex and the STN, pass from the motor and associative cortex through the dorsal area of STN; however, those from the limbic cortex pass through the anterior area of STN [33]. The results of the sweet spot analysis were inconsistent with the FDG-PET results. However, a previous study on non-human primates, using anterograde tracers, revealed overlapping projections from M1 to the dorsolateral STN and from the a

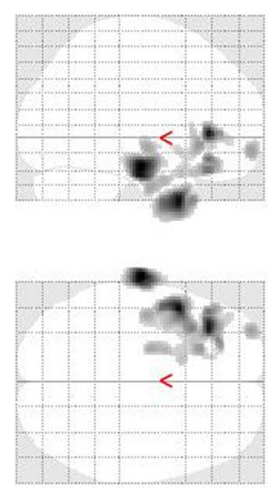

b
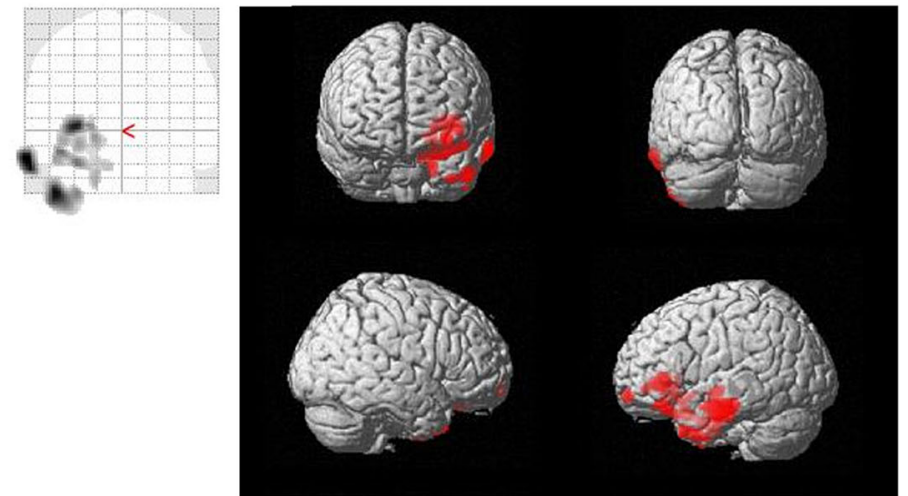

Fig. 3 Positive correlations between weight gain and brain metabolism detected using fluorodeoxyglucose-positron emission tomography were based on three orthogonal views (a) and a three-dimensional brain surface projection (b) 


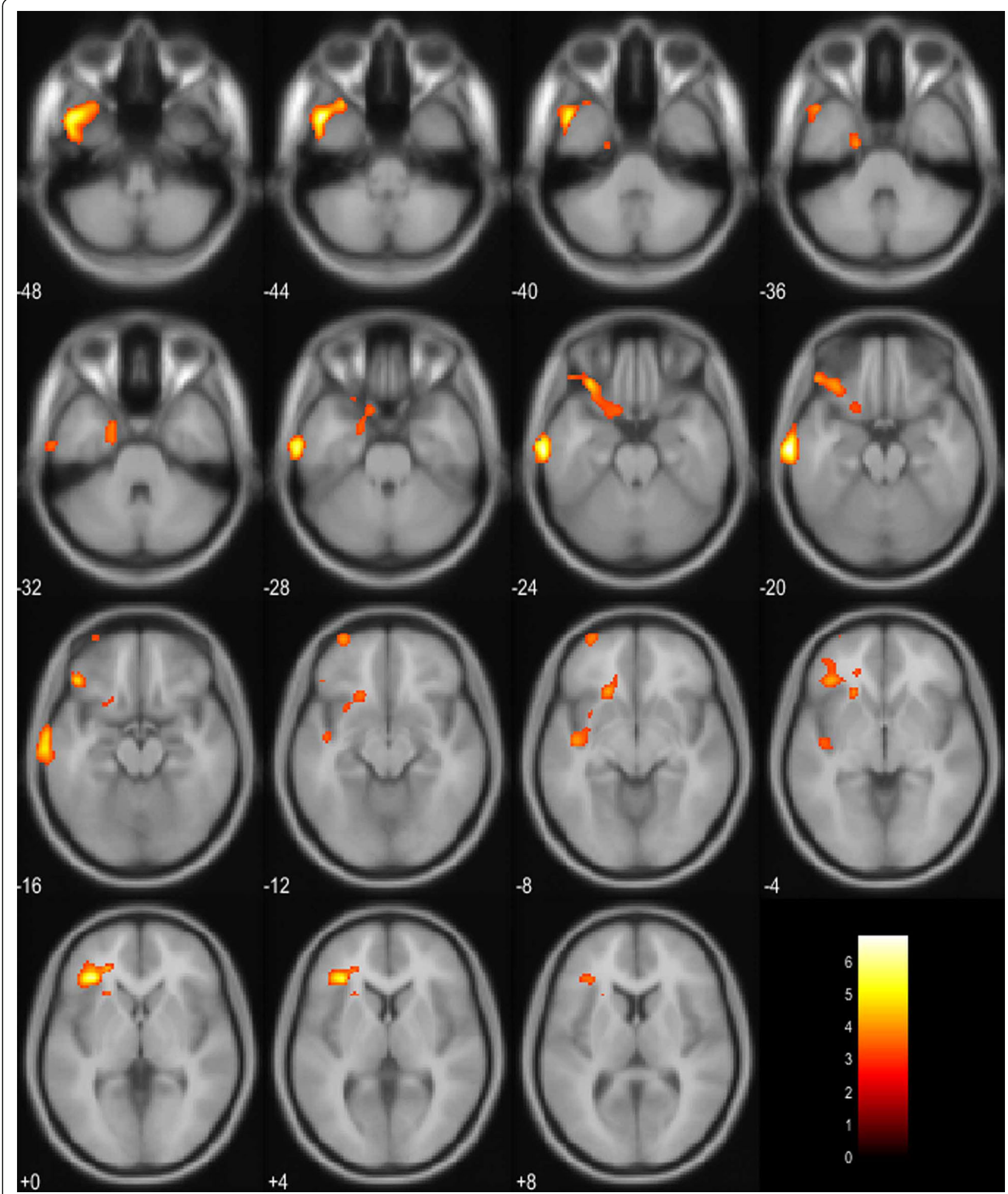

Fig. 4 Projection of voxels that show positive correlations between weight gain and increased brain metabolism on axial template brain slices. The yellow marked regions indicate areas with significantly positive correlation between weight gain and brain metabolism

prefrontal cortical areas to the anterior, ventral, and medial half of the STN [15]. Another study using diffusion tensor imaging found overlaps between the subregions within the STN, and their gradual transition into each other [18]. These findings suggest that neurons in each STN subregion receive multiple inputs and 
integrate information from different cortical regions. Stimulation to hyperdirect pathways passing the dorsal area of the STN may influence the integration of information from different cortical regions, and alter the activities of limbic cortex. A larger prospective study with correction for multiple comparisons is warranted to confirm this hypothesis.

We observed that WG was correlated with increased brain metabolism only on the left side. Several studies have also indicated that unilateral STN-DBS causes WG $[60,61]$, although the laterality of this relationship remains unclear. Further studies are warranted to determine whether right-side STN-DBS may influence metabolism in limbic and associative regions, and subsequently cause WG.

In this study, LEDD did not decrease significantly following STN-DBS. This may be linked to the fact that some patients were tremor dominant or experienced severe side effects with dopaminergic therapy. These patients were treated with a relatively low dose of dopaminergic medication and did not need a reduction in dopaminergic medication following DBS surgery. Indeed, the median LEDD value in this study was lower than that reported in previous studies. In addition, an aggressive reduction in dopaminergic medication following DBS may incur behavioral side effects, such as apathy and depression [62]. We reduced the medication dose carefully to prevent these behavioral side effects; this may be another reason why LEDD did not show any significant reduction following DBS surgery. Although a previous study reported significant correlation between WG and LEDD reduction [63], we could not find any correlation after multivariate regression analysis in this study. This may be attributed to the relatively small magnitude of LEDD reduction. However, future studies with larger sample sizes are needed to evaluate the effect of LEDD reduction on WG after STN-DBS.

This study has several limitations. First, we did not assess eating habits or daily food intake. Thus, future studies must confirm whether WG is caused by stimulation of the limbic area, which induces changes in eating behaviors, using preoperative and postoperative data on eating behaviors and food intake. Second, we did not assess hormonal factors or swallowing function, which could have confounded our analyses. Third, we obtained PET data only in an "off DBS" state after the surgery, owing to safety reasons. Therefore, it is difficult to attribute the changes in brain metabolism after DBS to the plasticity of the neural circuit or the washout process of therapeutic DBS. Fourth, we were not able to obtain a statistically significant model on multivariate regression analysis. This may be related to a relatively smaller number of patients compared to the number of independent variables. Further studies with a larger number of patients are required to confirm the correlation between active contact location and WG.

\section{Conclusions}

Although the mechanisms underlying WG following subthalamic deep brain stimulation are possibly multifactorial, our findings suggest that stimulation to the dorsal outside of the STN may lead to WG. Additionally, the metabolic changes in limbic and associative cortical regions following STN-DBS also correlated with WG. Stimulation to hyperdirect pathways passing through the dorsal area of the STN may influence the integration of information from different cortical regions and change the functions of the limbic and associative cortex. Further investigations are warranted to confirm this hypothesis by accurately assessing eating behaviors and food intake.

\section{Abbreviations}

BMl: Body mass index; CT: Computed tomography; FAB: Frontal Assessment Battery; LEDD: Levodopa-equivalent daily dose; MMSE: Mini Mental State Examination; MNI: Montreal Neurological Institute; MRI: Magnetic resonance imaging; PD: Parkinson's disease; PHQ-9: Patient Health Questionnaire-9; PSC: Percent signal change; UPDRS: Unified Parkinson's Disease Rating Scale; VTA: Volume of tissue activation; WG: Weight gain

\section{Acknowledgements \\ We thank Ms. Megumi Takeuchi for performing the neuropsychological examinations.}

\section{Authors' contributions}

Examination and treatment of Parkinson disease: K. Eguchi, S. Shirai, M. Matsushima, T. Kano, K. Yamazaki, S. Hamauchi, T. Sasamori, T. Seki, and Yabe I. PET data acquisition and analysis: K. Hiraka and T.

Shiga. Neuropsychological assessment: M. Otsuki. Drafting of the manuscript: K. Eguchi. Critical revision of the manuscript for important intellectual content: I. Yabe. Supervision: M. Kitagawa, K. Houkin, and H. Sasaki. All authors read and approved the final manuscript.

\section{Funding}

This work was supported in part by a Grant-in-Aid for the Research Committee of CNS Degenerative Diseases under Research on Measures for Intractable Diseases from the Ministry of Health, Welfare, and Labor, Japan [grant number 20FC1049]. The funder had no role in the study design, data collection, data analysis, data interpretation, or writing of the manuscript.

\section{Availability of data and materials}

The dataset(s) used and/or analyzed during the current study are available from the corresponding author on reasonable request.

\section{Declarations}

Ethics approval and consent to participate

This study was conducted following the 1964 Declaration of Helsinki and its later amendments and was approved by the institutional review board of Hokkaido University Hospital. All patients provided written informed consent prior to their inclusion in the study.

Consent for publication

Not applicable.

Competing interests

The authors declare that they have no competing interests. 


\section{Author details}

'Department of Neurology, Faculty of Medicine, Graduate School of Medicine, Hokkaido University, Kita 15, Nishi 7, Kita-ku, 060-8638 Sapporo, Japan. ${ }^{2}$ Department of Neurosurgery, Faculty of Medicine, Graduate School of Medicine, Hokkaido University, Kita 15, Nishi 7, Kita-ku, 060-8638 Sapporo, Japan. ${ }^{3}$ Department of Neurosurgery, Sapporo Azabu Neurosurgical Hospital, Kita 22, Higashi 1, Higashi-ku, 065-0022 Sapporo, Japan. ${ }^{4}$ Department of Diagnostic Imaging, Faculty of Medicine, Graduate School of Medicine, Hokkaido University, Kita 15, Nishi 7, Kita-ku, 060-8638 Sapporo, Japan. ${ }^{5}$ Sapporo Teishinkai Hospital, Kita 33, Higashi 1, Higashi-ku, 065-0033 Sapporo, Japan. ${ }^{6}$ Faculty of Health Sciences, Graduate School of Health Sciences, Hokkaido University, Kita 15, Nishi 7, Kita-ku, 060-8638 Sapporo, Japan. 'Department of Nuclear Medicine, Faculty of Medicine, Graduate School of Medicine, Hokkaido University, Kita 15, Nishi 7, Kita-ku, 060-8638 Sapporo, Japan.

\section{Received: 2 April 2021 Accepted: 1 September 2021}

\section{Published online: 13 September 2021}

\section{References}

1. Krack P, Batir A, Van Blercom N, Chabardes S, Fraix V, Ardouin C, et al. Fiveyear follow-up of bilateral stimulation of the subthalamic nucleus in advanced Parkinson's disease. N Engl J Med. 2003;349:1925-34.

2. Montaurier C, Morio B, Bannier S, Derost P, Arnaud P, Brandolini-Bunlon M, et al. Mechanisms of body weight gain in patients with Parkinson's disease after subthalamic stimulation. Brain. 2007:130:1808-18.

3. Jorgensen HU, Werdelin L, Lokkegaard A, Westerterp KR, Simonsen L. Freeliving energy expenditure reduced after deep brain stimulation surgery for Parkinson's disease: Decreased energy expenditure after STN-DBS surgery. Clin Physiol Funct Imaging. 2012;32:214-20.

4. Gironell A, Pascual-Sedano B, Otermin P, Kulisevsky J. Weight gain after functional surgery for Parkinsons disease. Neurologia. 2002;17:310-6.

5. Barichella M, Marczewska AM, Mariani C, Landi A, Vairo A, Pezzoli G. Body weight gain rate in patients with Parkinson's disease and deep brain stimulation. Mov Disord. 2003;18:1337-40.

6. Balestrino $R$, Baroncini D, Fichera $M$, Donofrio CA, Franzin A, Mortini $P$, et al. Weight gain after subthalamic nucleus deep brain stimulation in Parkinson's disease is influenced by dyskinesias' reduction and electrodes' position. Neurol Sci. 2017:38:2123-9.

7. Zahodne LB, Susatia F, Bowers D, Ong TL, Jacobson CE 4th, Okun MS, et al. Binge eating in Parkinson's disease: prevalence, correlates and the contribution of deep brain stimulation. J Neuropsychiatry Clin Neurosci. 2011;23:56-62.

8. Amami $P$, Dekker I, Piacentini S, Ferré F, Romito LM, Franzini A, et al. Impulse control behaviours in patients with Parkinson's disease after subthalamic deep brain stimulation: de novo cases and 3-year follow-up. J Neurol Neurosurg Psychiatry. 2015;86:562-4.

9. Guimarães J, Moura E, Vieira-Coelho MA, Garrett C. Weight variation before and after surgery in Parkinson's disease: a noradrenergic modulation? Mov Disord. 2012;27:1078-82.

10. Seifried C, Boehncke S, Heinzmann J, Baudrexel S, Weise L, Gasser T, et al. Diurnal variation of hypothalamic function and chronic subthalamic nucleus stimulation in Parkinson's disease. Neuroendocrinology. 2013;97:283-90.

11. Rieu I, Derost P, Ulla M, Marques A, Debilly B, De Chazeron I, et al. Body weight gain and deep brain stimulation. J Neurol Sci. 2011;310:267-70

12. Tripoliti E, Zrinzo L, Martinez-Torres I, Tisch S, Frost E, Borrell E, et al. Effects of contact location and voltage amplitude on speech and movement in bilateral subthalamic nucleus deep brain stimulation. Mov Disord. 2008;23: 2377-83.

13. Bot M, Schuurman PR, Odekerken VJJ, Verhagen R, Contarino FM, De Bie RMA, et al. Deep brain stimulation for Parkinson's disease: defining the optimal location within the subthalamic nucleus. J Neurol Neurosurg Psychiatry. 2018:89:493-8

14. Karachi C, Yelnik J, Tandé D, Tremblay L, Hirsch EC, François C. The pallidosubthalamic projection: an anatomical substrate for nonmotor functions of the subthalamic nucleus in primates. Mov Disord. 2005;20: 172-80.

15. Haynes WIA, Haber SN. The organization of prefrontal-subthalamic inputs in primates provides an anatomical substrate for both functional specificity and integration: implications for Basal Ganglia models and deep brain stimulation. J Neurosci. 2013;33:4804-14.
16. Lambert C, Zrinzo L, Nagy Z, Lutti A, Hariz M, Foltynie T, et al. Confirmation of functional zones within the human subthalamic nucleus: patterns of connectivity and sub-parcellation using diffusion weighted imaging. Neuroimage. 2012;60:83-94.

17. Accolla EA, Dukart J, Helms G, Weiskopf N, Kherif F, Lutti A, et al. Brain tissue properties differentiate between motor and limbic basal ganglia circuits. Hum Brain Mapp. 2014;35:5083-92.

18. Plantinga BR, Temel $Y$, Duchin $Y$, Uludağ $K$, Patriat $R$, Roebroeck $A$, et al. Individualized parcellation of the subthalamic nucleus in patients with Parkinson's disease with 7T MRI. Neuroimage. 2018;168:403-11.

19. Eisenstein SA, Koller JM, Black KD, Campbell MC, Lugar HM, Ushe M, et al. Functional anatomy of subthalamic nucleus stimulation in Parkinson disease: STN DBS Location and PD. Ann Neurol. 2014;76:279-95.

20. Akram H, Sotiropoulos SN, Jbabdi S, Georgiev D, Mahlknecht P, Hyam J, et al. Subthalamic deep brain stimulation sweet spots and hyperdirect cortical connectivity in Parkinson's disease. Neuroimage. 2017;158:332-45.

21. Gourisankar A, Eisenstein SA, Trapp NT, Koller JM, Campbell MC, Ushe M, et al. Mapping movement, mood, motivation and mentation in the subthalamic nucleus. R Soc Open Sci. 2018;5:171177.

22. Castrioto A, Lhommée E, Moro E, Krack P. Mood and behavioural effects of subthalamic stimulation in Parkinson's disease. Lancet Neurol. 2014;13:287-305.

23. Le Jeune F, Drapier D, Bourguignon A, Péron J, Mesbah H, Drapier S, et al. Subthalamic nucleus stimulation in Parkinson disease induces apathy: a PET study. Neurology. 2009;73:1746-51

24. Le Jeune F, Péron J, Biseul I, Fournier S, Sauleau P, Drapier S, et al. Subthalamic nucleus stimulation affects orbitofrontal cortex in facial emotion recognition: a PET study. Brain. 2008;131:1599-608.

25. Hughes AJ, Daniel SE, Kilford L, Lees AJ. Accuracy of clinical diagnosis of idiopathic Parkinson's disease: a clinico-pathological study of 100 cases. J Neurol Neurosurg Psychiatry. 1992;55:181-4.

26. Lang AE, Houeto J-L, Krack P, Kubu C, Lyons KE, Moro E, et al. Deep brain stimulation: preoperative issues. Mov Disord. 2006:21:S171-96.

27. Starkstein SE, Fedoroff JP, Price TR, Leiguarda R, Robinson RG. Apathy following cerebrovascular lesions. Stroke. 1993;24:1625-30.

28. Kroenke K, Spitzer RL, Williams JB. The PHQ-9: validity of a brief depression severity measure. J Gen Intern Med. 2001:16:606-13.

29. Tomlinson CL, Stowe R, Patel S, Rick C, Gray R, Clarke CE. Systematic review of levodopa dose equivalency reporting in Parkinson's disease. Mov Disord. 2010;25:2649-53.

30. Horn A, Kühn AA. Lead-DBS: A toolbox for deep brain stimulation electrode localizations and visualizations. Neuroimage. 2015;107:127-35.

31. Horn A, Li N, Dembek TA, Kappel A, Boulay C, Ewert S, et al. Lead-DBS v2: Towards a comprehensive pipeline for deep brain stimulation imaging. Neuroimage. 2019;184:293-316.

32. Avants BB, Epstein CL, Grossman M, Gee JC. Symmetric diffeomorphic image registration with cross-correlation: evaluating automated labeling of elderly and neurodegenerative brain. Med Image Anal. 2008;12:26-41.

33. Ewert S, Plettig P, Li N, Chakravarty MM, Collins DL, Herrington TM, et al. Toward defining deep brain stimulation targets in MNI space: a subcortical atlas based on multimodal MRI, histology and structural connectivity. Neuroimage. 2018;170:271-82.

34. Horn A, Reich M, Vorwerk J, Li N, Wenzel G, Fang Q, et al. Connectivity predicts deep brain stimulation outcome in Parkinson disease. Ann Neurol. 2017;82 1:67-78,

35. Treu S, Strange B, Oxenford S, Neumann WJ, Kühn A, Li N, et al. Deep brain stimulation: imaging on a group level. Neuroimage. 2020:219:117018.

36. Della Rosa PA, and the EADC-PET Consortium, Cerami C, Gallivanone F, Prestia A, Caroli A, et al. A standardized [18F]-FDG-PET template for spatial normalization in statistical parametric mapping of dementia. Neuroinformatics. 2014;12:575-93.

37. Bannier S, Montaurier C, Derost PP, Ulla M, Lemaire J-J, Boirie Y, et al. Overweight after deep brain stimulation of the subthalamic nucleus in Parkinson disease: long term follow-up. J Neurol Neurosurg Psychiatry. 2009:80:484-8.

38. Sauleau P, Le Jeune F, Drapier S, Houvenaghel J-F, Dondaine T, Haegelen C, et al. Weight gain following subthalamic nucleus deep brain stimulation: a PET study. Mov Disord. 2014;29:1781-7.

39. de Chazeron I, Pereira B, Chereau-Boudet I, Durif F, Lemaire JJ, Brousse G, et al. Impact of localisation of deep brain stimulation electrodes on motor and neurobehavioural outcomes in Parkinson's disease. J Neurol Neurosurg Psychiatry. 2016;87:758-66

40. Adamantidis A, de Lecea L. Sleep and metabolism: shared circuits, new connections. Trends Endocrinol Metab. 2008;19:362-70. 
41. Rossi PJ, Gunduz A, Okun MS. The subthalamic nucleus, limbic function, and impulse control. Neuropsychol Rev. 2015;25:398-410.

42. Espinosa-Parrilla J-F, Baunez C, Apicella P. Modulation of neuronal activity by reward identity in the monkey subthalamic nucleus. Eur J Neurosci. 2015;42:1705-17.

43. Barutca S, Turgut M, Meydan N, Ozsunar Y. Subthalamic nucleus tumor causing hyperphagia-case report. Neurol Med Chir (Tokyo). 2003:43:457-60.

44. Etemadifar M, Abtahi SH, Abtahi SM, Mirdamadi M, Sajjadi S, Golabbakhsh A, et al. Hemiballismus, hyperphagia, and behavioral changes following subthalamic infarct. Case Rep Med. 2012;2012:768580.

45. Aiello M, Eleopra R, Rumiati RI. Body weight and food intake in Parkinson's disease. A review of the association to non-motor symptoms. Appetite. 2015;84:204-11.

46. Ardouin C, Voon V, Worbe Y, Abouazar N, Czernecki V, Hosseini H, et al. Pathological gambling in Parkinson's disease improves on chronic subthalamic nucleus stimulation. Mov Disord. 2006;21:1941-6.

47. Kistner A, Lhommée E, Krack P. Mechanisms of body weight fluctuations in Parkinson's disease. Front Neurol. 2014;5:84.

48. Lim SY, O'Sullivan SS, Kotschet K, Gallagher DA, Lacey C, Lawrence AD, et al. Dopamine dysregulation syndrome, impulse control disorders and punding after deep brain stimulation surgery for Parkinson's disease. J Clin Neurosci. 2009;16:1148-52.

49. Witjas T, Baunez C, Henry JM, Delfini M, Regis J, Cherif AA, et al. Addiction in Parkinson's disease: impact of subthalamic nucleus deep brain stimulation. Mov Disord. 2005;20:1052-5.

50. Rothemund Y, Preuschhof C, Bohner G, Bauknecht H-C, Klingebiel R, Flor H, et al. Differential activation of the dorsal striatum by high-calorie visual food stimuli in obese individuals. Neuroimage. 2007;37:410-21.

51. Führer D, Zysset S, Stumvoll M. Brain activity in hunger and satiety: an exploratory visually stimulated FMRI study. Obesity (Silver Spring). 2008;16:945-50.

52. Killgore WDS, Young AD, Femia LA, Bogorodzki P, Rogowska J, YurgelunTodd DA. Cortical and limbic activation during viewing of high- versus lowcalorie foods. Neuroimage. 2003;19:1381-94.

53. Siep N, Roefs A, Roebroeck A, Havermans R, Bonte ML, Jansen A. Hunger is the best spice: an fMRI study of the effects of attention, hunger and calorie content on food reward processing in the amygdala and orbitofrontal cortex. Behav Brain Res. 2009:198:149-58.

54. Goldstone AP, Prechtl de Hernandez CG, Beaver JD, Muhammed K, Croese C, Bell G, et al. Fasting biases brain reward systems towards high-calorie foods. Eur J Neurosci. 2009;30:1625-35.

55. Wallner-Liebmann S, Koschutnig K, Reishofer G, Sorantin E, Blaschitz B, Kruschitz $R$, et al. Insulin and hippocampus activation in response to images of high-calorie food in normal weight and obese adolescents. Obesity (Silver Spring). 2010;18:1552-7.

56. Wang G-J, Volkow ND, Telang F, Jayne M, Ma J, Rao M, et al. Exposure to appetitive food stimuli markedly activates the human brain. Neuroimage. 2004;21:1790-7.

57. Carnell S, Gibson C, Benson L, Ochner CN, Geliebter A. Neuroimaging and obesity: current knowledge and future directions. Obes Rev. 2012;13:43-56.

58. Tataranni PA, DelParigi A. Functional neuroimaging: a new generation of human brain studies in obesity research. Obes Rev. 2003;4:229-38.

59. Abizaid A, Horvath TL. Brain circuits regulating energy homeostasis. Regul Pept. 2008; 149:3-10

60. Walker HC, Lyerly M, Cutter G, Hagood J, Stover NP, Guthrie SL, et al. Weight changes associated with unilateral STN DBS and advanced PD. Parkinsonism Relat Disord. 2009;15:709-11.

61. Lee EM, Kurundkar A, Cutter GR, Huang H, Guthrie BL, Watts RL, et al. Comparison of weight changes following unilateral and staged bilateral STN DBS for advanced PD. Brain Behav. 2011;1:12-8.

62. Rossi M, Bruno V, Arena J, Cammarota Á, Merello M. Challenges in PD patient management after DBS: a pragmatic review. Mov Disord Clin Pract. 2018:53:246-54.

63. Aiello M, Eleopra R, Foroni F, Rinaldo S, Rumiati RI. Weight gain after STNDBS: The role of reward sensitivity and impulsivity. Cortex. 2017;92:150-61.

\section{Publisher's Note}

Springer Nature remains neutral with regard to jurisdictional claims in published maps and institutional affiliations.

\section{Ready to submit your research? Choose BMC and benefit from:}

- fast, convenient online submission

- thorough peer review by experienced researchers in your field

- rapid publication on acceptance

- support for research data, including large and complex data types

- gold Open Access which fosters wider collaboration and increased citations

- maximum visibility for your research: over $100 \mathrm{M}$ website views per year

At BMC, research is always in progress.

Learn more biomedcentral.com/submissions 